\title{
Casual Information Visualization: Depictions of Data in Everyday Life
}

\author{
Zachary Pousman, John T. Stasko, Member, IEEE, and Michael Mateas
}

\begin{abstract}
Information visualization has often focused on providing deep insight for expert user populations and on techniques for amplifying cognition through complicated interactive visual models. This paper proposes a new subdomain for infovis research that complements the focus on analytic tasks and expert use. Instead of work-related and analytically driven infovis, we propose Casual Information Visualization (or Casual Infovis) as a complement to more traditional infovis domains. Traditional infovis systems, techniques, and methods do not easily lend themselves to the broad range of user populations, from expert to novices, or from work tasks to more everyday situations. We propose definitions, perspectives, and research directions for further investigations of this emerging subfield. These perspectives build from ambient information visualization [32], social visualization, and also from artistic work that visualizes information [41]. We seek to provide a perspective on infovis that integrates these research agendas under a coherent vocabulary and framework for design. We enumerate the following contributions. First, we demonstrate how blurry the boundary of infovis is by examining systems that exhibit many of the putative properties of infovis systems, but perhaps would not be considered so. Second, we explore the notion of insight and how, instead of a monolithic definition of insight, there may be multiple types, each with particular characteristics. Third, we discuss design challenges for systems intended for casual audiences. Finally we conclude with challenges for system evaluation in this emerging subfield.
\end{abstract}

Index Terms-Casual information visualization, ambient infovis, social infovis, editorial, design, evaluation.

\section{INTRODUCTION}

Much of the work in information visualization assumes a population of expert users who have knowledge and experience in analyzing problems in specific domains. Workers in widely varying domains from finance to government to journalism use information visualization tools to explore data, generate, refine and test hypotheses, and ultimately to produce insight. This user population of information workers and information analysts, combined with their needs and tasks and the environments in which these systems are used have had a substantial impact on the tools.

Information analysts employ infovis systems frequently, but not constantly. Analysts select an infovis tool when they enter into work phases where data understanding and hypothesis generation is important. These phases or episodes of use are focused and can last from a few minutes to a few hours. Other tasks take up much of the rest of an analyst's typical day. Outside of an episode with an infovis tool, an information worker's time is spent doing other tasks, such as digesting written reports, brainstorming, and meeting with colleagues. We call this usage pattern of infovis "episodic", characterized by its duration, intensity, and goal-orientation.

Some information visualization systems are however not designed for these user populations and these work situations. Instead, the systems are designed for more casual uses and without the same degree of task focus. For example, many people use digital tools to view and manage data that is important to them, from email to music to photo collections. Systems for visualizing this personal data, including Photomesa [7], which people use to view and manage thousands of images, certainly have some of the properties of an infovis system.

- Zachary Pousman is with the School of Interactive Computing and the GVU Center at the Georgia Institute of Technology, E-mail: zach@cc.gatech.edu.

- John Stasko is with the School of Interactive Computing and the GVU Center at the Georgia Institute of Technology, E-mail: stasko@cc.gatech.edu.

- Michael Mateas is with the University of California, Santa Cruz, E-mail: michaelm@cs.ucsc.edu.

Manuscript received 31 March 2007; accepted 1 August 2007; posted online 27 October 2007.

For information on obtaining reprints of this article, please send e-mail to: tvcg@computer.org.
Are these types of tools really infovis systems? The question arises, where are the limits of infovis with respect to the everyday uses of computational artifacts.

Card, Mackinlay, and Shneiderman define information visualization broadly: infovis is the use of computers to interactively amplify cognition, using visual representations [10]. Therefore if we take this as our definition, systems must be computer-based, interactive, provide visual representations, and most importantly, amplify cognition.

In the sections that follow, we examine four categories of systems that appear to fit this definition but also likely are not commonly considered as a part of traditional infovis and thus have not been analyzed or explained in the vocabulary of infovis research. The systems can be thought of as positioned near the boundary between infovis and other domains including ubiquitous computing, design, and art. The infovis community has a long history of transforming data into visualizations to support thinking, and using the vocabulary and techniques of infovis may be helpful to the researchers working at these boundaries.

\section{Centers and Margins}

Agre notes that many engineering fields have underlying metaphors that shape the practice of researchers [2]. He notes that what is central to the metaphorical description becomes "core", pushing other aspects or properties to the periphery or margin. By exploring the margins, insight can be reached that can both show new ways forward for the field, but can also reflect back on the central metaphors themselves.

Our goal in this paper is to reflect on systems that are pushed to the edge of the infovis domain by two related metaphors and assumptions. The first is an assumption about human beings as information processors [11]. Under this very well established view of human capabilities, systems are designed to highlight our rational processes, and push non-rational parts of human nature to the margins. Second, traditional infovis systems have long been thought of in work terms, as designed for information workers, and with the attendant delineated tasks that come from work life. The boundary that we explore in this paper is amongst systems that are not designed for work tasks. Instead, they are, in a word, more casual.

We note that there are other boundaries of infovis. The boundary between scientific visualization and infovis has been explored by Tory and Moller [37] who note ways that discrete and continuous data and the attribute types form a design space that contains both infovis and scientific visualization. 


\section{Method and Plan}

We characterize a set of systems as positioned at the boundary between infovis and other domains. Our method consisted of identifying the systems that exhibit at least the property that they represent information visually to change or facilitate a user's thinking, much like core infovis. Others modify one or more of the properties of Card et. al. , or eliminate them altogether.

Our analysis is adopted from prototype theory, an alternative to the set-theoretic model of classification of items into categories [23]. As such, our analysis does not focus on providing a clearly delineated and binary boundary between those systems which are core to infovis and which are on the periphery. Our goal is not to create a taxonomy.

Instead, we subscribe to ideas from prototype theory including Centrality Gradience, that some examples of a class are better representations of that category than others, and Membership Gradience, that membership in classes is better characterized as a matter of degree, not of necessary and sufficient conditions. We do not find it profitable to make some final determination on each system as either falling "inside" or "outside" of infovis. Instead, by embracing the edge cases and determining vocabulary to describe them, we can shed light on the underlying metaphors of infovis as a discipline, and show new research questions and open areas ripe for growth of the field.

In the next sections we introduce a set of exemplars that are edge cases to traditional infovis research and we organize them into categories that frame smaller subareas of research. Then, based on these exemplars, we present a definition of casual information visualization. In later sections we note changes to design questions and begin the work of presenting design issues and evaluation strategies for Casual Infovis researchers. We posit that design issues and evaluation questions on the edges of infovis can apply back to the more traditional systems in the field as well, enriching not just the margins, but the center as well.

\section{Infovis at the Edges}

\section{Ambient Infovis}

Ambient information systems [28], systems that sit in peripheral locations and provide abstract depictions of data can qualify under the broadest definition of infovis - they visualize information. Skog et. al. introduced the term Ambient Infovis as they worked on Informative Art, a set of systems that map data into subtle, changing portraits of data [32]. Informative Art uses inspiration from modern art works (including Piet Mondrian) to convey data such as bus departure times and weather data (see Figure 1A). Colors, shapes, and positions of objects in an electronic painting change to reflect updates in the data being conveyed. Ambient Infovis trades reduced user interaction for an increase in aesthetic emphasis [32].

Some other exemplars of ambient information systems are the Ambient Orb [4], InfoCanvas [36], and Sideshow (now called "Sidebar" and integrated into Microsoft Windows Vista) [9] (see Figure 1). The Ambient Orb maps stock data (or weather data) into the color of the glowing orb, fading slowly from green when stocks are rising to red when stocks are dropping. The InfoCanvas consolidates information from many sources (stocks, weather, traffic, airline prices, email) into a single abstract painting, where each element in the image can be tied to data. The Sideshow system is a peripheral information bar for a desktop computer that uses widgets to display similar personal information. Can these systems be thought of as infovis?

One reason that these systems may not be considered infovis is the diminished interactive capabilities they exhibit. Though simple interactions are possible (users of InfoCanvas can brush across picture elements to show their underlying data), exploring of the data by changing representations is often beyond the capability of ambient infovis.

The Ambient Orb only represents a single number (the percentage change in the stock market at a that time). What is the lower bound of information needed to "amplify cognition"? Another question that ambient infovis makes plain is the requirement to represent data. In some ambient infovis systems, including parts of Sideshow (see Figure 1d), data is left in its raw form. Core infovis systems re-represent data,
A)

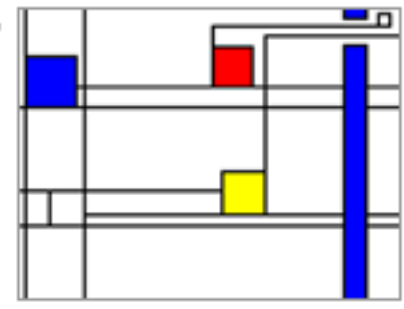

C)

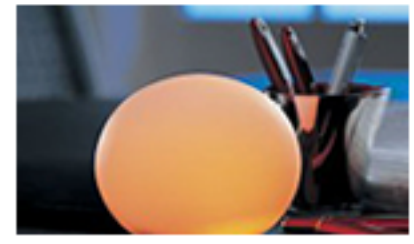

D)

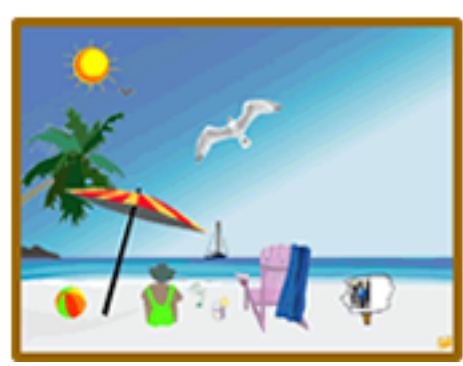

B)

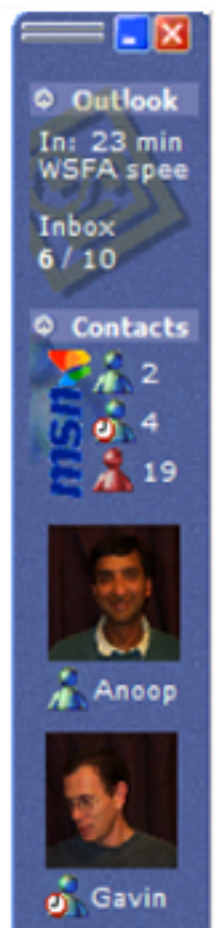

Fig. 1. A) Informative Art B) Sideshow (Image (C)2002 ACM, Inc. Reproduced by permission.) C) Ambient Orb D) InfoCanvas

transforming the data from numerical or textual symbols, or other raw forms, into some new format. One may ask whether these widgets are sufficiently "visual" enough to be infovis?

\section{Social Infovis}

Social information surrounds us, and takes forms that lend themselves to being visualized. Articles are collaboratively written and images and songs are shared, sampled, and remixed. Technology support for tagging of digital artifacts has created spaces for collaborative web bookmarking (e.g. del.icio.us), news (e.g. digg.com), and even public space (e.g. plazes.com, yellowarrow.org). Visualizations of social processes, social networks, and social situations have become another emerging and exciting domain for infovis researchers.

Social infovis systems span a range of data sources, representation techniques, and usage. PeopleGarden [45] is a system to provide an overview of an online community. The system maps users to flowers in a garden, and each flower's attributes such as height, petal color, and number of petals represent a user's posting history (see Figure 2A).

Vizster [22] creates node-link diagrams of social networks on the internet website Friendster ([20]). Instead of starting with a view of the entire friendster network (hundreds of thousands of nodes), Vizster begins with a view of the user herself at the center and only her friends displayed (see Figure 2C). The authors call this user-centric approach "ego-centric" and claim it has advantages over the more traditional (but often implicit) data-centric view.

Ego-centricity also appears in Viegas' Themail system [39] that visualizes the huge volume of email that many of us struggle to make sense of. Themail performs word counts on the header and body information of an email box, and then creates columns of words with size and coloration based on the number of instances (which is a measure of perceived importance) (see Figure 2B). In a user study, participants found this visualization helpful in reflecting on past events and recollecting their email usage.

Finally, we note a visualization of shared web bookmarks that has become ubiquitous on sites that allow bookmarking and "tagging", a visualization of user-generated taxonomies. These visualizations, called Tag Clouds (e.g. [15]), are used to form a gestalt view of the 
A)

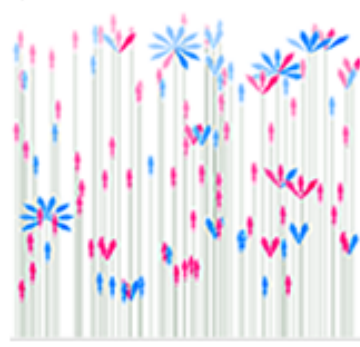

B)

C)

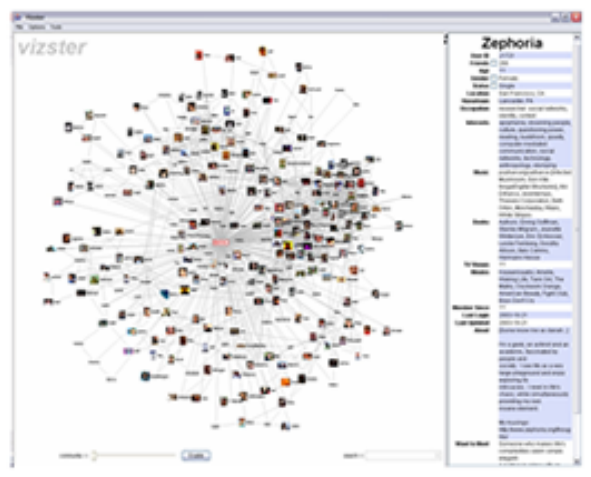

Fig. 2. A) PeopleGarden B) Themail C) Vizster (Images (C) 1999, 2006 ACM, Inc. Reproduced by permission.)

interests of a single user or group of users.

\section{Artistic Infovis}

Yet another emerging area of information visualization work are systems that are works of data-driven art. These systems too display data, and transform that data into visual representations, some of which are interactive. Viegas and Wattenberg have coined the term artistic infovis to describe the way that infovis techniques might apply to art works [41]. In our view, these systems have the explicit goal of challenging preconceptions of data and representation. In the vocabulary of the art world, they "problematize" our everyday conceptions.

However, these systems are different from more traditional infovis systems in one important respect. Traditional infovis systems maintain a functionalist perspective, in that they are designed to be helpful for a particular set of analytic tasks. In contrast, data-driven artworks may challenge some of our notions of visualization and computer-mediated understanding, our notions of what constitutes data, and may even question our ideas about the infrastructure of computer systems. In everyday use, infrastructure is invisible and often goes unexamined [34]. Artistic infovis systems can make computer networks, algorithms, and data itself objects for reflection.

Because these systems may evoke curiosity, puzzlement, or even frustration, they depart from just being aesthetically pleasing or welldesigned. Of course, as art, many of them are beautiful. But these systems are artistic in their orientation and framing, not just merely elegant or beautiful, like the ambient information systems above (which retain some of the functional focus of traditional infovis systems). We include further examples below that may highlight their differences from traditional infovis systems.

Two pieces of data-driven art reflect the art gallery scene back to the viewers and have some of the characteristics of infovis. Artifacts of the Presence Era [40], by Viegas and Donath creates evocative views of the flow of human traffic in the art gallery. The system records video and then stacks and warps the video based on the ambient sound level inside the gallery (see Figure 3A). The work calls into question the viewer/artifact relationship, since the viewer is an active participant in what the art looks like. He or she can experiment with movements and vocalizations to see how it affects the system's output.

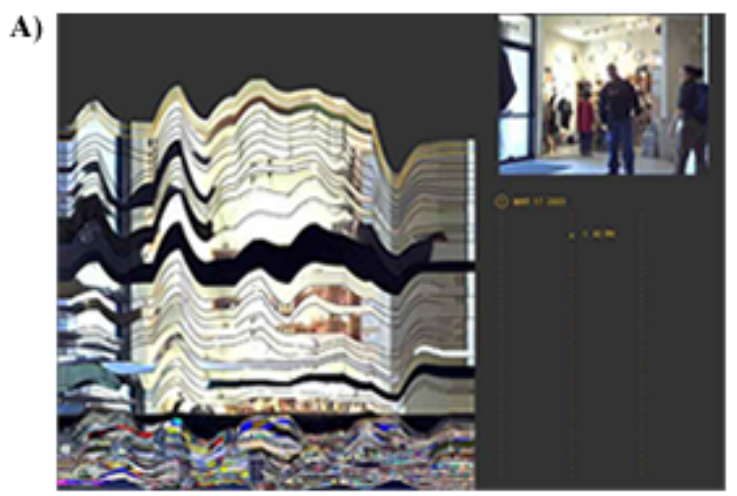

B)
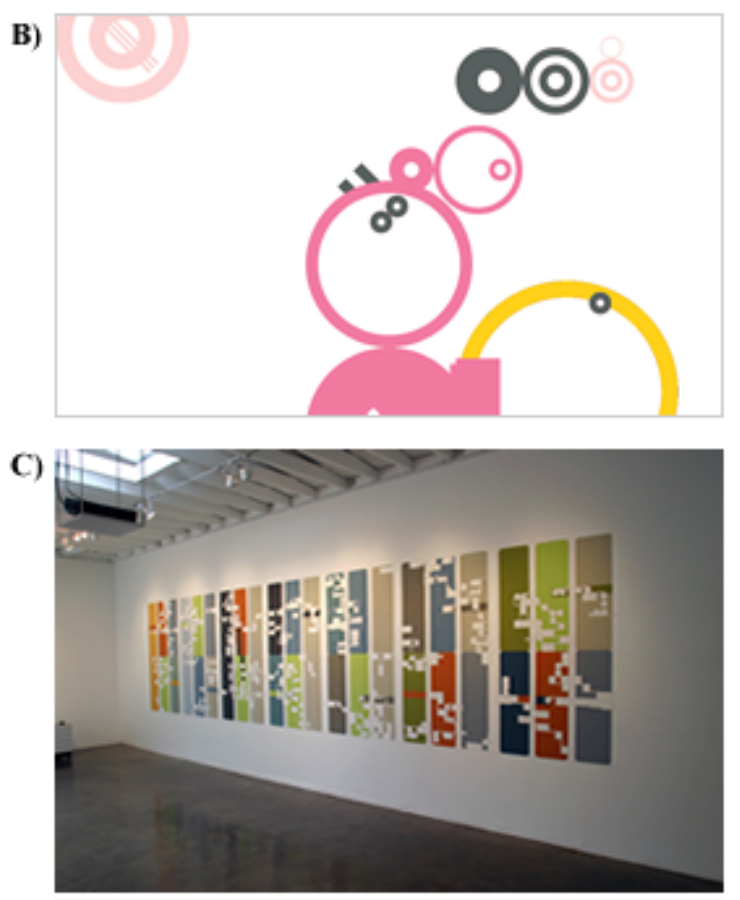

D)

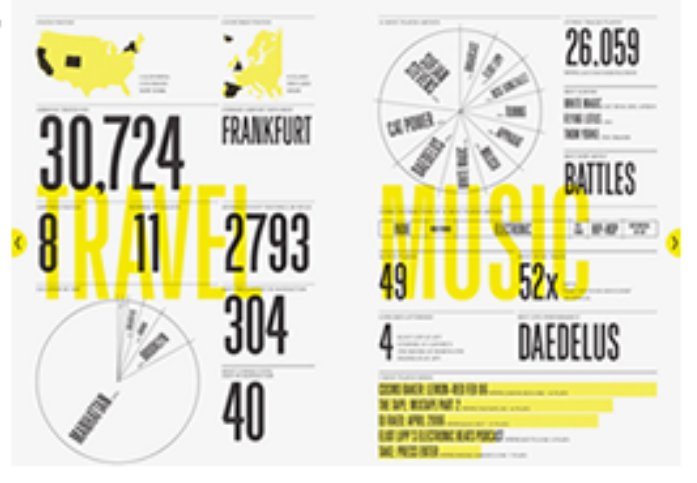

Fig. 3. A) Artifacts of the Presence Era B) Tableau Machine C) Visitor.Files D) Annual Report 2006

Sorting [44] by Wignell is another art piece that reflects the gallery back to the viewer. The system takes live video and performs an ordering of all of the pixels by color intensity. This creates bands of color that are evocative of the viewers, but not explicit. This work brings questions about "data" into focus; what counts as data? Is video, after it has been sorted into a new form still meaningful?

Romero et. al. designed a system, the Tableau Machine [30], that works as a "social mirror" of home life, allowing insight into the pat- 
terns, rhythms, and routines of domestic space and modern family life. The system senses individuals moving about the spaces of the home using cameras and characterizes the movement that people (and pets) generate. The system measures changed pixels every frame, giving a proxy of household "activity", its location and time. From this raw activity measure, the system computes proxy measures of togetherness, and social flow. These three measures, taken together, give something of the flavor or mood of the house.

Every few minutes the system produces abstract paintings on a large screen. These modernist images are made from primitive shapes whose layout, size, and color is influenced by the data. The the relationship between data and the output is, however, not discrete; no particular shape represents any person or place in the home. Instead, the data changes the compositional rules, so busy times in the home produce very full and busy collages with energetic color palettes, and calm moments in the home create more delicate collages, with fewer shapes and subdued color palettes (see Figure 3B).

This system is intentionally ambiguous, open to interpretation by members of the home (and their guests). This is obviously different from core infovis systems, which most often have a single "correct" reading of the visual output, and a mapping that ties one piece of data to a single visual representation.

Ray's Visitor.Files [29] is an installation that consists of large paintings along with video and notebooks of her data. Ray spent weeks at a busy street corner in Los Angeles and recorded detailed and structured observations of the activity there, including the movement of vehicles and pedestrians, clouds in the sky and the temperature. She then produced paintings of the data that are in essence information visualizations, painted by hand but based on exacting specification and mapping (see Figure 3C).

Visitor.Files instantiates an information visualization algorithm in a human substrate. The human is the sensor, recording the data from the world; the aggregator, counting instances based on her pre-defined rules; and finally, she is the representation engine, painting from a set procedure to display the data. This work expounds on notions of artist as interpreter, and artist as cog in a larger system of production. For our discussion, it may spark reflections about the attenuation of an infovis algorithm through time, and also about algorithms being instantiated in human activity, rather than computer code.

Lastly we note Feltron's 2006 Annual Report [18], an annually produced pamphlet by Feltron, a graphic designer. Feltron takes advantage of the digital traces created by personal activities and compiles the data into a series of info graphics and tables (see Figure 3D). He notes how many meals he ate, where they were eaten, which songs and musical artists were listened to with great frequency, and other seeming inanities of modern life. The pamphlet, through simple visualizations, gives a snapshot image of Feltron's life and the descriptive statistics provide a telling portrayal of what Feltron does and what he values.

Taken together, these systems beg us to confront the notion of infovis as an amplifier of cognition. Are these art systems making our thinking better? Surely seeing an everyday situation, such as an afternoon around the house (e.g. Tableau Machine) or a trip to an art gallery (e.g. Artifacts of the Presence Era), with a fresh perspective is valuable. This is clearly not the same kind of amplified cognition that genomics researchers or financial analysts come to, but there is more to cognition than merely insight for work tasks. These systems may trigger us to reflect on our lives, our worlds, and the hidden properties of sensors and representations (e.g. Visitor.Files). Lastly, these systems may help us in meta-cognition, thinking about our own thinking.

\section{Other Infovis Edge Cases}

The preceding three sub-domains are quite active and and are full of examples of edge cases. However, there are many other systems that may also be considered edge cases that do not seem to fit into the categories of ambient infovis, social infovis, or artistic infovis. These edge cases range from online tools, such as Smartmoney's Map of the Market [42], or Digg.com's "Stack", "Swarm", and "Arc" visualizations [16], to less task-focused visualizations of weblog posts, such as "The Dumpster" [17].
A)

B)
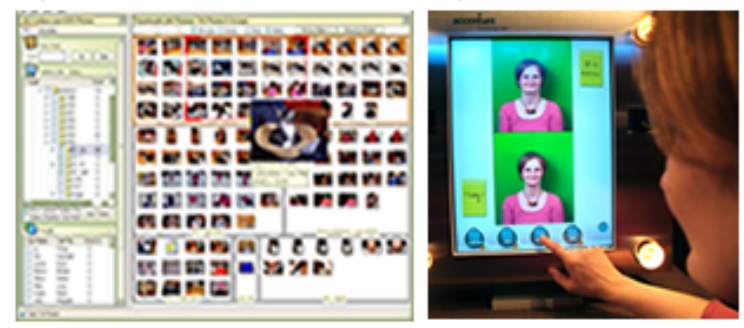

C)

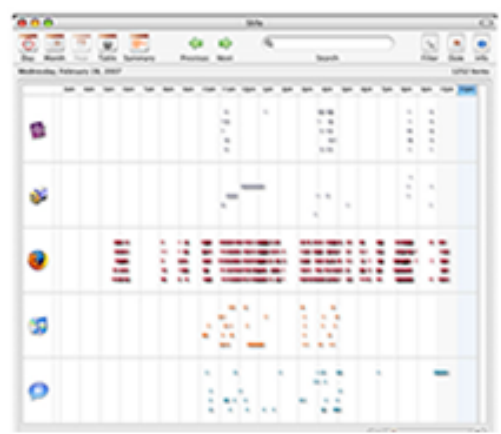

Fig. 4. A) Photomesa Image (C)2001 ACM, Inc. Reproduced by permission.)B) Persuasive Mirror C) Slife

The area of personal infovis, information visualizations of personal data contain a number of systems of note. Photomesa [7], one of our early examples of an edge case, does not seem to fit cleanly into any of the preceding categories. Instead it is a visualization of personal data that uses space-filling treemaps to display a personal photo collection. A commercial product, Slife [33], produces time-line visualizations of each application used on a Apple Mac computers. Slife creates a portrait of computer usage over days, weeks, and months, allowing reflection on the portion of one's day spent using web browsers, versus email applications, versus word processors.

Another edge category can be described as persuasive visualization. Captology is the study of computers as persuasive things [19]. Computers can be to persuade individuals to recycle more, exercise more, or to make other healthy lifestyle choices. Many of Fogg's proposals for systems in this area use visualizations to make visible results of our decisions. Andrés de Valle and Opalach built a prototype of a Persuasive Mirror [14], that would monitor a user's diet and display the future (heavier, thinner, unchanged) when the user looked in a video "mirror."

These examples, and the examples from artistic, ambient, and social infovis might be seen as far from the systems that make up the core of infovis. And not all of them may truly be information visualizations. But we claim that it is worth knowing where infovis ends and other disciplines such as art, ubiquitous computing, and persuasive technology begin. Looking at the margins of the field may provide insights into problems and opportunities at the core of the field, and vocabulary that we may use to describe these edge cases may be applicable to the entirety of information visualization.

So our work here is to embrace these edge cases, and to ask seriously what properties move a system to the edges of the class. Our plan is to dissect the systems in terms of their design goals and to articulate a vocabulary for discussing them.

\section{Casual information Visualization}

The systems presented above may be considered to be far from the core set of infovis systems, and may seem quite alien. However, they do fit the definition of being visual representations of data: they do visualize information. In our view the Ambient Infovis, Social Infovis, and Artistic Infovis systems, as well as other new domains and uses of visualization point to a complement to traditional infovis that we call Casual Information Visualization (Casual Infovis). All of these 
sub-domains share the same relationship to more traditional infovis research, that of center and margin. Though each sub-domain has its own character, we propose Casual Infovis as an umbrella term that reframes ambient, social, and artistic infovis, as well other edge cases as a part of, but different from, more traditional infovis systems and techniques. We note four differences between traditional infovis systems and Casual Infovis.

* User Population: The user population is enlarged to include a wide spectrum of users from experts to novices. Users are not necessarily expert in analytic thinking, nor are they required to be experts at reading visualizations.

* Usage Pattern: Usage expands past work, to focus on other parts of life. Systems are intended for usage that is momentary and repeatable (over weeks and months), or contemplative (a long moment at an art gallery).

* Data type: The data is typically personally important and relevant, as opposed to work-motivated. This means that a user's relationship to the data is often a more tightly coupled one.

* Insight: We propose that the kinds of insight that Casual Infovis may support are different from more traditional systems. We suggest that developers are interested in providing insight about data that is not analytical, but instead of a different sort.

Thus, we define: Casual Infovis is the use of computer mediated tools to depict personally meaningful information in visual ways that support everyday users in both everyday work and non-work situations. Below, we expand upon this definition through more careful consideration of the four characteristics. We do note that not every Casual Infovis system has each criterion shifted completely to the margin. For instance, an ambient visualization of work tasks may still be considered a Casual Infovis system, even though the data is work motivated, if it shifts intended insights to reflection.

Furthermore, Casual Infovis systems are not defined by the visualization technique used and, in fact, may use core infovis techniques such as a treemaps, node-link graph diagrams, or scatterplots. We claim that Casual Infovis systems are those that include many (if not all) of the design attributes above. Data, users, context of use, and insights sought create a totality of experience that is different from that of core infovis systems.

\section{User Population and Usage Pattern}

The users of casual information visualizations are of a wider variety than the target user populations of more central infovis tools. The users are drawn from a wide spectrum as regards their level of expertise with analytic problem solving. Casual Infovis systems do not require users to be "information analysts" and therefore expect that users do not always have deeply analytical ways of thinking and working. They may also not be experts in understanding complicated graphs and charts of data. As we have seen in the case of the "tag cloud" social infovis, the text is only modified on a small number of attributes (size, color, and position). This may well be near the limit of a social visualization intended for wide audiences.

Developers also build Casual Infovis systems for a different usage pattern than that found in traditional infovis systems. We characterize usage for more central infovis systems as "episodic," with users diving deeply into exploring the dataset for a period of hours, with few interruptions. Casual Infovis usage patterns often consist of fleeting moments of inspection over long periods of time. For example, self reports from an in situ evaluation of the InfoCanvas found that users continued to glance at the InfoCanvas display over the entire month that it was deployed in their office [35]. Similarly, viewers typically glance at the Map of the Market for a few moments and perhaps explore a stock or two, but they do not dwell on the display for hours.

Other Casual Infovis experiences can serve as support for contemplation and reflection, especially those that use the conventions of art to allow new ways of looking at things. Though the use of an infovis system at an art gallery is usually measured in minutes, the new perspective can stay with users for a long while.

\section{Data}

The character of the data in Casual Infovis systems often shifts from the work domain to the personal domain. Because personal data is by definition personal, the data contain nuances and subtleties that may not be present in work data. The data is not merely the photos or messages, but additionally the meanings that these tokens conveya beautiful hike in the woods, a wedding day, or a child's first steps. The meaning of these images is delicate, often unspoken and different for different users in different situations. We call this distinction one between the denotative meaning (what the picture depicts plainly, such as a couple on a beach) versus that of the connotative meaning (what the picture represents, such as a honeymoon).

In Photomesa [7], users may find insights in patterns of the photos they see that might not be seen by a disinterested third party. These meanings come from the mind of the collection's owner as she browses, not just from the data itself, as is more common in the case of traditional infovis. van Wijk notes that domain experts can get more from an infovis system than novices, since they bring their understanding to their use of the system [38]. Is personal knowledge the same kind of knowledge that we have about work tasks? We feel that data about our lives leads to different kinds of knowledge than domain knowledge. This is a result of both the type of data, and what we do with that data-how we make it meaningful. We return to this point later when discussing insight.

Of course, personal collections of data are not entirely full of gems. Many are mundane moments, bicycles that we might have photographed for sale, or, in the case of email, the endless march of coordination emails such as "Will you get the dry cleaning on the way home?" or "Lunch at noon?". However, the separation is often not so clearly delineated. A blurry photo of an unimportant lunch may be the only picture one has of a particular sweater, or a particular haircut, or a particular group of friends. This may be one's only remembrance of that group. Those picture elements could, in the right circumstance, provide just the right juxtaposition with our current haircuts or our current social situation to be helpful in some reflection. Instead of being easier in personal data sets to separate the important from the unimportant, or the interesting from the mundane, it may well turn out to be more difficult.

\section{Insight}

Another change, and one we feel is more fundamental than those discussed previously, is the change in insight at the edges of infovis. Casual Infovis shifts the goals of the systems that are built; system designers focus on creating insights that are different from the design goals of traditional infovis systems. If infovis is intended, according to Card, Mackinlay, and Shneiderman, to amplify cognition and to provide insight, what does this insight consist of? What may help to define Casual Infovis systems is to explore the shift in these edge case's sense of providing insight. We take up this hypothesis in the next section, by proposing that insight is multi-faceted, with Casual Infovis systems providing insights that are different in kind and not just different in degree from those provided by more core infovis systems.

\section{From INFORMATION TO INSIGHT}

A long-standing catch phrase, if not a definition, of information visualization is that the systems turn information into insight. This transformative power is an important one, because infovis is not merely pretty pictures, but instead must result in some change to the user's understanding.

As van Wijk notes, an infovis system can, at its most simple, be a visualization of "a single bit," the smallest quanta of information [38]. However, the relationship between information and insight is more complicated than a simple linear relation, and the insight that results could be large or small. Similarly, a system that displays millions of 
bits of information (in the information-theoretic sense) may produce large or small insights.

Insight may come from focused work, or it may emerge without a fixed or explicit task. Insight can be intentionally sought out by an individual, but it can also come about from merely going about one's daily life; reading the newspaper, and taking part in the activities of work and play.

We take insight to be something complex and heterogenous, not simplistic or monolithic. Insight is a term that cuts to the very core of cognition, understanding, and learning, and particular theoretical models of cognition influence our views of just what understanding might be. That said, we have tried to cast a wide net through the infovis domain, without a focus on particular mental models, and we propose the following set of different types of insight. These different kinds of insigtht expand on Saraiya et. al. 's [31] seven properties of insight that include the observation itself, its value to the field (in their case genomics), its correctness, and its breadth versus depth.

We move beyond Saraiya et. al. 's properties to investigate observations about data that are less explicitly analytical. Humans are more than "information processors"; they are citizens, parents, and dreamers. Casual Infovis systems focus on awareness insights, social insights, and also reflective insights. We note that the types of insight are not mutually exclusive, so a particular insight might have components of both analytic insight and social insight.

\section{Analytic insight}

Analytic insight is the most traditional sense of insight that we find supported in visualization systems. The focus on analytic insight is one of the hallmarks of traditional infovis research.

Analytic insights come from exploratory analysis, extrapolation, and consist in the large or small eureka moments where a body of data comes into focus for a user. Wehrend and Lewis identified 11 low-level tasks which can result in an analytic insight for a user [43] Amar and Stasko have investigated two gaps where infovis tools are lacking for analytic insights, those on the way to identifying useful relationships and comparisons, termed worldview gaps, and those gaps of rationale and confidence, termed the rationale gap [3]. Identifying and bridging these gaps allow lower-level insights to become a part of higher-level insights, such as those in learning a topic, predicting future states, and discovery of causality.

However, Casual Infovis researchers and their boundary systems provide other kinds of insight that complement this more traditional view. By broadening our view of insight, Casual Infovis can help us to open new areas for information visualization research.

\section{Awareness insight}

Awareness insight is the insight that comes from maintaining awareness of a particular data stream. Maintaining awareness can give a sense of fluctuations in the data and its shifting patterns, even though awareness insight does not require that the user come to some crystalized conclusion. Instead the insight can come from the subtly useful information that, even if a user is only barely conscious of it, he or she knows and can apply in the appropriate situation. These kinds of insights, such as having a grasp of the weather forecast for tomorrow, or news stories for cocktail parties can give users wide views (if often shallow) of data streams.

Staying aware of information does not help in some analytic task, but instead helps people communicate socially, keep on top of cultural trends and memes, and make connections between domains in informal ways. Maintaining awareness is a low-intensity activity, and as such, the insights that come from these activities (and these systems) are less clear-cut than insights that come from deep and intense analysis. These insights may not rise to the stature of analytic insights, but awareness insights keep us aware of the outside world and information that may impact us in the future.

Ambient visualization systems often produce awareness insights about data that is either user-selected, or selected by designers to be personally relevant to a community or type of individual.

\section{Social insight}

Social insight is insight about social networks, social situations, and social life. Social insight is the sense of understanding of a social group and one's place in it. Social insight is also not as task focused as analytic insight, because these conclusions are contingent and subject to revision and reinterpretation.

These insights can be ideas that the user already possesses to a greater or lesser extent. In their use of some social vis tool, users can confirm these suspicions or preconceived notions. Things like "This person is very active (in this community)" or "This person has a lot of friends" are often, if given time to consider it for a moment, probably obvious to members of that community. Confirmation is valuable, as are the times when a hunch is disproved by the tool in question. These reflections can serve as resources for informal social conversation, as well as informal changes to work processes. So, while not having a particularly analytical character, gaining insight into social workings can open new hypotheses, invite reflection, and even change social or individual behavior.

\section{Reflective insight}

Reflective insight is insight about oneself, the world, and one's place in it. These insights come from data also, but in a less constrained way. Systems that display and present information (in this case, information may be a much larger body of knowledge than in the previous kinds of insight) can provide evidence and fodder for reflecting on and contemplating one's personal and idiosyncratic thoughts. One of the ways that reflection can be prompted is by defamiliarization, making the mundane and everyday strange [8]. This is often accomplished by taking a novel perspective or getting "distance" from the situation or activity. Ideas from defamiliarization have worked their way into HCI, and we feel that they are appropriate for Casual Infovis as well.

Artistic infovis systems often use defamiliarization to help users make reflective insights. The Tableau Machine, for example, does not attempt to depict the rhythms and routines in a home as a person might, but instead as another kind of sentient being. This change in perspective means that the system might confuse a household fight from a party, since both are moments of heightened activity and energy. Therefore generated images in both situations could appear very similar. But the system's confusion might lead to interest, reflection, and conversation amongst the members of the household. Defamiliarization of the home makes the visualization stronger, even as it changes the notion of what it means for a system to provide insight.

\section{Design Challenges for Casual Infovis}

In the same way that information visualization can crystallize the relationship between variables in an analysis task at work, information visualization techniques and methods may be adapted to home life, to personal data sources, and to the social groups in which we work, play, and live. People leave many digital traces as they go about their daily lives because of the wealth of computer systems we interact with. Casual information visualization is different from more core infovis research. Different users, different data, and providing different kinds of insight require modifications to the design process and tradeoffs that must be dealt with by systems designers. Developers cannot merely apply techniques from infovis wholesale to Casual Infovis systems.

We claim that developers of Casual Infovis face the following challenges. First, they trade "utilitarian" design goals for a broader set of design goals that we term "useful." Second, we introduce some ways for developers to think about long-term usefulness and meaning as they design. Finally, we suggest that developers can improve systems if they understand the idiosyncratic, private (and often unspoken), and delicate nature of people's lives outside of focused episodes of work. We suggest ethnographic techniques as a potential aid for designers.

Casual Infovis moves outside of the utilitarian concerns of more traditional infovis work. Core infovis systems can be helpful to users thinking through problems of analysis in deep, episodic, and goaloriented ways. Casual Infovis research moves into domains that are less task focused, such as managing and understanding one's home, or 
reflecting on one's photo collection. We characterize traditional infovis systems as designed to be helpful by being "utilitarian", which we contrast with a more general sense of tools being "useful" for Casual Infovis. In core infovis, a system should have a tool-like ability to do work to display data, uncover trends and outliers, and generate hypotheses. Casual Infovis systems are useful artifacts that are helpful for providing representations of data, but without a clear task focus.

A term that is further from the core of infovis research that can describe systems that are non-utilitarian is "pleasing", which to us has a connotation of being delightful without regard whatsoever for their utility. This term applies to some of the artistic infovis systems presented, since they make no claims about being useful or utilitarian. A system that is pleasing might make a user smile just by seeing it. Opposed to this are ambient infovis systems and ambient information systems that retain a sense of being useful and not merely decoration. This terminology is related to Norman's notions of visceral appeal (emotionally or aesthetically focused), behavioral appeal (task focused), and reflective appeal (higher cognitive processes) [26].

Design of Casual Infovis is also concerned with the meanings that systems convey. Since much of the data depicted is personal, the visualizations are often meaningful to users, even if a stranger might find the visualization merely curious. Meaningfulness can also be enhanced by ambiguity. Ambiguity can be a resource for design, opening spaces for personal stories, and for personal meaning [5]. The traditional view of information visualization is that systems should be efficient and effective, that they should show the data and nothing more. Casual Infovis systems can be (but are not required to be) ambiguous or equivocal in their depiction of data. By this, we mean that there can exist multiple "correct" interpretations of the underlying data, instead of a single correspondence between data and display. This space supports reflection on the true meaning of the data.

Finally, because Casual Infovis systems need to be integrated into the lives of users, their particular rhythms and routines, more information is needed by designers. Design methods that seek to uncover these rhythms, including participatory design, ethnographically inspired design, and autobiographical design have been suggested to help with these concerns [13]. Designers need to be attuned to the ways that people live, their concerns, their internal conceptions. This can effect what data matters, where potential users might already do these reflective and contemplative activities.

\section{Evaluation Challenges for Casual infovis}

Casual Infovis changes how system builders should evaluate their creations. Infovis systems are hard to evaluate in general how can a researcher know whether a certain conclusion would have been reached without the tool, or whether a tool is helpful in generating insights or amplifying cognition? But Casual Infovis systems can make matters even more complicated. First, the systems are less productivity focused, so even determining what to measure is difficult. Second systems are meant to provide the multiple varieties of insights, and these are softer than analytic insights, which makes them difficult for evaluators to gauge. Lastly, systems are also designed for more casual usage patterns, so traditional laboratory evaluations may be inappropriate for the long-term benefits that casual systems intend to bring about.

Saraiya et. al. address this question to some degree with their introduction of "Insight-based evaluation" which is a technique for understanding the number, type, and importance of the insights that a tool can bring [31]. Their evaluation method was performed in a controlled laboratory experiment, so it is of limited value for the longitudinal evaluations that Casual Infovis may require. Plaisant notes a similar set of challenges of infovis evaluation and contrasts four large categories of evaluation found in the infovis system literature [27]. She enumerates two types of laboratory evaluations, either of a system's components or comparative evaluations between tools, as well as usability evaluations and case studies of longitudinal deployments.

We note some distinctions between evaluating Casual Infovis systems and more traditional systems. Different usage patterns, different data, and different system goals mean new evaluation methods are needed. Evaluations for casual infovis can borrow from ubiquitous computing and other fields that push evaluation out into the world, instead of evaluating system use in the laboratory [1]. Measurement of casual infovis systems take place over a longer time horizon, because users need time to move from being distracted by a system, or excited merely because a system is novel, and into more normal and everyday use. Gaver notes that mixed methods and multiple perspectives have uncovered successes and failures about casual systems that display information about life [21].

Evaluation of casual infovis systems is made even more difficult due to the kinds of insights that designers attempt to convey. In particular, Casual Infovis systems provide insight into the daily flux of information, the evolving social life of work, home, and play, or insight into a mundane activity that is made unfamiliar or made strange. All of these insights are not tied to productivity metrics like efficiency and effectiveness. Nor are they driven by, or the product of, work tasks. In this sense, these Casual Infovis systems are beyond productivity [24].

One would like to retain the generalizable evaluation methods used in assessing more traditional infovis as we move to Casual Infovis systems. Increasingly, evaluations of infovis systems focus on user satisfaction in addition to measuring usability and efficiency metrics. This has often included narratives and explanations from study participants via think-aloud protocols or post-study open-ended interviews. The results of these query techniques can give more credence to timed or otherwise objective criteria as participants note a particular system feature, or a pattern that they notice in the data. But more work in this area is needed to assess systems whose usage is frequent but momentary. Insights into the data, moments of reflection, and observations of changes in work practices (or home routines) are difficult to capture, as well as being difficult for study participants to remember. One approach that shows some promise is the experience sampling method (ESM), borrowed from HCI and ubicomp research [6, 12]. This method allows sampling at regular or random intervals, where participants might be asked to fill out a small survey, or recollect on the past few days in a more freeform manner. Over a period of weeks and months, patterns of usage, problems, and common sentiments begin to reveal themselves.

These evaluation goals, and the methods that we may adapt or invent to support measuring them, will be helpful for Casual Infovis researches. In addition, these methods may actually improve the scope and quality of evaluations for more traditional infovis systems as well. The techniques that help system builders deepen the user narratives, move toward longitudinal studies of how systems come to be utilized, and a concern with the experience and felt life of a technology artifact all can be applied to the "center" of infovis, not just at the margins.

\section{Conclusions and Future Directions}

Casual Infovis complements more traditional infovis research. Casual Infovis systems share many of the properties of more traditional systems, but with an increased focus on activities that are less task driven, data sets that are personally meaningful, and built for a wider set of audiences. To some, our work in defining Casual Infovis may be overargued, with all of the systems presented here being unremarkable examples of infovis. But we feel that the differences are worthy of note, because they point to new challenges and questions. We claim that widening our perspective regarding insight to include those insights that are not analytic in nature will help developers explore this space.

This paper opens the conversation about Casual Infovis systems. We suggest preliminary terminology and understandings of how these systems work, and note the many interesting research challenges of designing Casual Infovis systems and evaluating their successes in helping less analytically minded user groups to make sense of the large and growing dataset that their digital traces leave behind. How can we design systems whose highest aims are not focused on productivity, but instead on notions of usefulness, enjoyment, and reflection?

Casual Infovis is, in our view, larger than merely the combination of social infovis, ambient infovis, and some artistic visualizations. There are other potentially fruitful domains for Ccasual infovis researchers to explore. Some future directions that we think are worthy of note are systems for personal (and family) finance, since household expen- 
ditures have rhythms and routines that people may want to track, understand, and reflect upon. Household energy use is another place where data is available, but often invisible. Casual Infovis can make reflecting on energy usage deeper and more interesting. Also we note that exercise and diet choices that is increasingly technology mediated. The Nike+ system [25] for tracking running and walking behavior is a popular data source for individuals to track their exercise progress. A final domain that is ripe for Casual Infovis is the increasingly common practice of digital travelogues that include text, images, and video.

Understanding the edge cases of infovis has brought into focus some of the underlying notions of task focus and information processing models that infovis researchers have used. We certainly do not feel that these notions are outmoded and useless. But knowing for which kinds of human endeavors they work can help developers as they build new systems. We hope to push the field forward using the particulars of Casual Infovis, but we hope that our results, evaluation techniques, and design guidelines will be more widely applicable, and can be helpful to developers of more traditional infovis techniques as well as Casual Infovis developers.

\section{ACKNOWLEDGEMENTS}

The authors wish to thank J. Clawson, C. Plaue, R. Amar, and K. Boehner for their comments on drafts of this paper. This work was supported in part by a grant from the National Science Foundation IIS-0414667.

\section{REFERENCES}

[1] G. D. Abowd and E. D. Mynatt. Charting past, present, and future research in ubiquitous computing. ACM Transactions Computer-Human Interaction, 7(1):29-58, 2000.

[2] P. Agre. Computation and Human Experience. Oxford University Press, 1997.

[3] R. Amar and J. Stasko. Knowledge precepts for design and evaluation of information visualizations. IEEE Transactions on Visualization and Computer Graphics (TVCG), 11(4):432-442, 2005.

[4] Ambient Devices, Inc. Ambient Orb. Retrived February 12, 2007 from http://www. ambientdevices.com/cat/orb/orborder. html.

[5] P. Aoki and A. Woodruff. Making space for stories: Abiguity in the design of personal communication systems. In Proceedings of CHI, 2005.

[6] L. Barrett and D. Barrett. An introduction to computerized experience sampling in psychology. Social Science Computer Review, 19(2):175$185,2001$.

[7] B. Bederson. Photomesa: a zoomable image browser using quantum treemaps and bubblemaps. In Proceedings of UIST 2001, pages 71-80, New York, NY, USA, 2001. ACM Press. (C)2002 ACM, Inc. Reproduced by Permission.

[8] G. Bell, M. Blythe, and P. Sengers. Making by making strange: Defamiliarization and the design of domestic technologies. ACM Trans. Comput.-Hum. Interact., 12(2):149-173, 2005.

[9] J. Cadiz, G. Venolia, G. Jancke, and A. Gupta. Designing and deploying an information awareness interface. In Proceedings of CSCW, New York, NY, 2002. ACM Press. (C)2002 ACM, Inc. Reproduced by permission.

[10] S. K. Card, J. Mackinlay, and B. Shneiderman. Readings in Information Visualization, Using Visualization to Think, chapter 1, pages 1-34. Morgan Kaufmann, 1999.

[11] S. K. Card, A. Newell, and T. P. Moran. The Psychology of HumanComputer Interaction. Lawrence Erlbaum Associates, Inc., Mahwah, NJ, USA, 1983

[12] S. Consolvo and M. Walker. Using the experience sampling method to evaluate ubicomp applications. IEEE Pervasive Computing Mobile and Ubiquitous Systems, 2(2):24-31, April 2003.

[13] A. Crabtree. Ethnography in participatory design. In R. Chatfield, S. Kuhn, and M. Muller, editors, Participatory Design Conference, pages 93-105, Seattle, Washington, USA, Nov. 1998.

[14] A. C. de Valle and A. Opalach. The persuasive mirror. In Proceedings of Persuasive 2006, May 2006.

[15] Del.icio.us. Popular Tags Cloud. Retrived March 22, 2007 from http: //del.icio.us/tags.

[16] Digg Labs. Retrieved July 8, 2007 from http:// labs.digg.com/.
[17] The Dumpster. Retrived July 8, 2007 from http://artport. whitney.org/commissions/thedumpster/.

[18] N. Feltron. Annual Report 2006. Retrived March 22, 2007 from http: //feltron.com/06report \_index.html.

[19] B. J. Fogg. Persuasive computing. In Proceedings of CHI 1998, 1998.

[20] Friendster. Retrived March 20, 2007 from http://www. friendster.com.

[21] W. Gaver, A. Boucher, S. Pennington, and B. Walker. Evaluating technologies for ludic engagement. In Proceedings of $\mathrm{CHI}$, Extended $\mathrm{Ab}$ stracts, 2005.

[22] J. Heer and danah boyd. Vizster: Visualizing online social networks. In Proceedings of IEEE Infovis, pages 33-40, 2005.

[23] G. Lakoff. Women, Fire, and Dangerous Things. University Of Chicago Press, April 1990.

[24] W. J. Mitchell, A. S. Inouye, and M. S. Blumenthal, editors. Beyond Productivity: Information Technology, Innovation, and Creativity. National Academy Press, 2003.

[25] Nike+ System. Retrived March 24, 2007 from http://www.nike. com/nikeplus/.

[26] D. A. Norman. Emotional Design: Why We Love (Or Hate) Everyday Things. Basic Books, January 2004.

[27] C. Plaisant. The challenge of information visualization evaluation. In Proceedings of AVI 2004, pages 109-116, 2004.

[28] Z. Pousman and J. Stasko. A taxonomy of ambient information systems: Four patterns of design. In Proceedings of Advanced Visual Interfaces, pages 67-74, May 2006.

[29] C. Ray. Visitor.Files. Retrived March 22, 2007 from http: //glowlab.com/lab2/artist_project.php?project_ id=9\\&artist_id=5.

[30] M. Romero and M. Mateas. A preliminary investigation of alien presence. In Proceedings of HCII 2005, 2005.

[31] P. Saraiya, C. North, and K. Duca. An insight-based methodology for evaluating bioinformatics visualizations. IEEE Transactions on Visualization and Computer Graphics, 11(4):443-456, July 2005.

[32] T. Skog, S. Ljunglblad, and L. E. Holmquist. Between aesthetics and utility: Designing ambient information visualization. In Proceedings of IEEE Infovis, pages 233-240, 2003.

[33] Slife Labs. Retrived March 20, 2007 from http://www . slifelabs.com/slife/features.

[34] S. L. Star. The ethnography of infrastructure. American Behavioral Scientist, 43(3):377-391, 1999.

[35] J. Stasko, D. McColgin, T. Miller, C. Plaue, and Z. Pousman. Evaluating the infocanvas peripheral awareness system: A longitudinal, in situ study. Technical Report GIT-GVU-05-08, Georgia Institute of Technology, March 2005.

[36] J. Stasko, T. Miller, Z. Pousman, C. Plaue, and O. Ullah. Personalized peripheral information awareness through information art. In Proceedings of Ubicomp 2004, pages 18-35, 2004.

[37] M. Tory and T. Moller. Rethinking visualizations: A high-level taxonomy. In Proceedings of IEEE Infovis, pages 151-158, 2004.

[38] J. J. van Wijk. Views on visualization. IEEE Transactions on Visualization and Computer Graphics (TVCG), 12(4):421-432, July/August 2006.

[39] F. Viegas, S. Golder, and J. Donath. Visualizing email content: Portraying relationships from conversational histories. In Proceedings of $\mathrm{CHI}$, New York, NY, USA, 2006. ACM Press. (C)2006 ACM, Inc. Reproduced by permission.

[40] F. Viegas, E. Perry, E. Howe, and J. Donath. Artifacts of the presence era: Using information visualization to create an evocative souvenir. In Proceedings of IEEE Infovis, pages 105-111, 2004.

[41] F. Viegas and M. Wattenberg. Artistic data visualization: Beyond visual analytics. In Proceedings of HCII 2007 (in press), July 2007.

[42] M. Wattenberg. Visualizing the stock market. In CHI '99: $\mathrm{CHI}$ extended abstracts on Human factors in computing systems, pages 188-189, New York, NY, USA, 1999. ACM Press.

[43] S. Wehrend and C. Lewis. A problem-oriented classification of visualization techniques. In Proceedings of IEEE Infovis, pages 139-143, 1990.

[44] A. Wignell. Sorting. Retrived February 2, 2007 from http://www . annawignell.com.

[45] R. Xiong and J. Donath. Peoplegarden: creating data portraits for users. In Proceedings of UIST (Symposium on User interface software and technology), pages 37-44, New York, NY, USA, 1999. ACM Press. (c)1999 ACM, Inc. Reproduced by permission. 